\title{
Relativistic ab initio study on PtF and HePtF $\uparrow$
}

\author{
Wenli Zou, Yang Liu and James E. Boggs* \\ Received 2nd November 2009, Accepted 14th December 2009 \\ First published as an Advance Article on the web 15th January 2010 \\ DOI: 10.1039/b922850k
}

The electronic structures and spectroscopic constants of the first three low-lying electronic states $(\Omega=$ $1 / 2,3 / 2$, and $5 / 2$ ) of the linear HePtF complex were investigated by highly accurate relativistic ab initio methods, in which the spin-orbit coupling was taken into account, and compared with the results of PtF. It shows that the complex is significantly different from the typical van der Waals systems because of short He-Pt bond distances $(1.80 \sim 1.87 \AA)$, large He-Pt stretching frequencies $\left(500 \sim 600 \mathrm{~cm}^{-1}\right)$, considerable binding energies (1400 2500 $\mathrm{cm}^{-1}$ with corrections), and a small electron transfer from helium (about 0.06 ). However, the topological analysis of the electron density distribution indicates that there is strong van der Waals interaction in the He-Pt bond instead of weak covalent one.

\section{Introduction}

Since xenon hexafluoroplatinate was experimentally prepared in $1962,{ }^{1}$ many neutral rare-gas compounds have been found. ${ }^{2}$ However, for the compounds of $\mathrm{He}$ and $\mathrm{Ne}$, nothing is known experimentally. Nevertheless, some neutral complexes of helium were theoretically predicted in the literature to be stable or metastable ( $c f$. Ref. ${ }^{3}$ for summary). Most of them belong to the so-called "strong van der Waals complexes". For these molecules, the calculated binding energies (BE) of helium only amount to a few kcal mol${ }^{-1}\left(1000 \sim 2000 \mathrm{~cm}^{-1}\right)$, and the topological analysis of the electron density distribution shows that there is no covalent bond formed on helium. ${ }^{3}$ The first example of a strong van der Waals complex is $\mathrm{HeBeO}{ }^{4}$

In a recent paper, we reported that there are considerable interactions between helium and metal atoms in $\mathrm{HeCuF}$ and $\mathrm{HeAuF}$ with the binding energies of 1632 and $1543 \mathrm{~cm}^{-1}$, respectively. ${ }^{3}$ Inspired by these two species, we expected that $\mathrm{HeNiF}$ and $\mathrm{HePtF}$ should also be metastable. In this paper we report studies of $\mathrm{HePtF}$ using high-level ab initio methods. HeNiF is not discussed here because of the strong static-correlations in the nickel atom, ${ }^{5,6}$ although our preparatory computations show that the binding energy of its ground state $(\Omega=3 / 2)$ is about $1600 \mathrm{~cm}^{-1}$.

\section{Computational details}

To account for the scalar relativistic effects, relativistic effective core potentials (RECPs) were used for $\mathrm{Pt}^{7}$ and $\mathrm{F}^{8}$ to replace the inner 60 and 2 core electrons, respectively. The valence basis of $\mathrm{Pt}$ was cc-pwCV5Z-PP, ${ }^{7}$ i.e., $(18 \mathrm{~s} 15 \mathrm{p} 13 \mathrm{~d} 5 \mathrm{f} 4 \mathrm{~g} 3 \mathrm{~h} 2 \mathrm{i}) /[9 \mathrm{~s} 9 \mathrm{p} 8 \mathrm{~d} 5 \mathrm{f} 4 \mathrm{~g} 3 \mathrm{~h} 2 \mathrm{i}]$. The exponents of the valence basis of $F$ were taken from allelectron cc-pV5Z 9 by deleting the first five steep s-functions, and were recontracted at the internally contracted multi-reference configuration interaction with singles and doubles (MRCI) ${ }^{10}$ level of theory, leading to $(9 \mathrm{~s} 8 \mathrm{p} 4 \mathrm{~d} 3 \mathrm{f} 2 \mathrm{~g} 1 \mathrm{~h}) /[5 \mathrm{~s} 5 \mathrm{p} 4 \mathrm{~d} 3 \mathrm{f} 2 \mathrm{~g} 1 \mathrm{~h}]$. For He, the

Institute for Theoretical Chemistry, Chemistry and Biochemistry Department, The University of Texas at Austin, Austin, Texas 78712-0165, USA. E-mail: james.boggs@mail.utexas.edu

$\dagger$ Dedicated to Professor David W. H. Rankin on the occasion of his retirement. standard cc-pV5Z basis set was used. ${ }^{11}$ These basis sets have 5-zeta quality and are abbreviated later as V5Z. In order to approximate the complete basis set limit via complete basis set extrapolation (CBSE) ${ }^{12}$ the corresponding basis sets with 4-zeta quality were used as well (denoted as VQZ).

Two kinds of calculations were performed in our research for the three low-lying scalar electronic states, i.e. $,{ }^{2} \Sigma^{+},{ }^{2} \Pi$, and ${ }^{2} \Delta$. In the multi-reference calculations, $\mathrm{PtF}$ and $\mathrm{HePtF}$ were computed with state-averaged CASSCF followed by MRCI with Davidson's cluster correction (MRCI+Q) ${ }^{10}$ There were 9 active molecular orbitals corresponding to Pt 5d6s and F 2p, whereas the Pt 5s5p, $\mathrm{F} 2 \mathrm{~s}$, and He $1 \mathrm{~s}$ orbitals were always doubly occupied and correlated. In the single-reference calculations, the restricted openshell Hartree-Fock based spin-unrestricted open-shell CCSD and $\operatorname{CCSD}(\mathrm{T})$ methods ${ }^{13,14}$ were used, and all the electrons except the ones replaced by RECPs were correlated, as in MRCI+Q.

Unlike the computations of $\mathrm{HeCuF}$ and $\mathrm{HeAuF}$, the spinorbit coupling (SOC) could not be neglected for $\mathrm{HePtF}$ and PtF. SOC was treated via the state-interaction approach ${ }^{15}$ at the CASSCF level, where the diagonal elements of the spin-orbit matrix calculated with spin-orbit RECP were later replaced by the MRCI+Q, CCSD, or CCSD(T) energies. From ${ }^{2} \Sigma^{+},{ }^{2} \Pi$, and ${ }^{2} \Delta$, five $\Omega$ states could be derived, i.e., (I) $1 / 2$, (I) $3 / 2$, (I) $5 / 2$, (II) $1 / 2$, and (II) $3 / 2$ with an energy gap of about $7000 \mathrm{~cm}^{-1}$ between the former three and the latter two spinor states. The cases of (II) $1 / 2$ and (II) $3 / 2$ are very complex since they cross with other higher excited states. In this research we focus only on the first three states.

At the minimum of each $\Omega$ state optimized with numerical gradients, the mass weighted second derivative matrix was numerically constructed for ${ }^{195} \mathrm{Pt}^{19} \mathrm{~F}$ or ${ }^{4} \mathrm{He}^{195} \mathrm{Pt}^{19} \mathrm{~F}$ by hand in order to make maximum use of the symmetry and minimize the number of displacement coordinates. The matrix elements were obtained by the 3-point finite difference scheme with a displacement of $0.01 \AA$. Then, the harmonic vibrational frequencies were obtained by diagonalizing the matrix. The infrared (IR) intensities were computed from the dipole moment derivative matrix at the CASSCF level of theory.

All the $a b$ initio calculations were performed using the MOLPRO program. ${ }^{16}$ 
Table 1 Adiabatic excitation energies $\left(T_{\mathrm{e}}\right)$, equilibrium bond distances $\left(\mathrm{R}_{\mathrm{Pt} \mathrm{F}}\right.$ and $\left.\mathrm{R}_{\mathrm{He}-\mathrm{Pt}}\right)$, binding energies $(\mathrm{BE})$, and vibrational frequencies $\left(v_{1}, v_{2}\right.$, and $v_{3}$ ) for $\mathrm{PtF}$ and $\mathrm{HePtF}$

\begin{tabular}{|c|c|c|c|c|c|c|c|c|c|}
\hline \multirow[b]{2}{*}{ State } & \multirow[b]{2}{*}{ Method } & \multirow[b]{2}{*}{$\mathrm{T}_{\mathrm{e}} / \mathrm{cm}^{-1}$} & \multirow[b]{2}{*}{$\mathrm{R}_{\mathrm{Pt}-\mathrm{F}} / \AA$} & \multirow[b]{2}{*}{$\mathrm{R}_{\mathrm{He}-\mathrm{Pt}} / \AA$} & \multirow[b]{2}{*}{$\mathrm{BE}^{a} / \mathrm{cm}^{-1}$} & \multicolumn{3}{|c|}{ Frequency ${ }^{b} / \mathrm{cm}^{-1}$} & \multirow[b]{2}{*}{ Components ${ }^{c}(\%)$} \\
\hline & & & & & & $v_{1}$ & $v_{2}$ & $v_{3}$ & \\
\hline \multicolumn{10}{|l|}{$\mathrm{PtF}$} \\
\hline \multirow[t]{4}{*}{$1 / 2$} & $\mathrm{MRCI}+\mathrm{Q}$ & 0 & 1.895 & & & & & 587 & ${ }^{2} \Sigma^{+}(69)+{ }^{2} \Pi(31)$ \\
\hline & CCSD & 0 & 1.894 & & & & & & ${ }^{2} \Sigma^{+}(69)+{ }^{2} \Pi(31)$ \\
\hline & $\operatorname{CCSD}(\mathrm{T})$ & 177 & 1.891 & & & & & 590 & ${ }^{2} \Sigma^{+}(66)+{ }^{2} \Pi(34)$ \\
\hline & $\mathrm{DFT}^{d}$ & 855 & 1.894 & & & & & 597 & \\
\hline \multirow[t]{5}{*}{$3 / 2$} & MRCI+Q & 205 & 1.878 & & & & & 590 & ${ }^{2} \Pi(83)+{ }^{2} \Delta(17)$ \\
\hline & CCSD & 111 & 1.878 & & & & & & ${ }^{2} \Pi(82)+{ }^{2} \Delta(18)$ \\
\hline & $\operatorname{CCSD}(\mathrm{T})$ & 0 & 1.874 & & & & & 593 & ${ }^{2} \Pi(83)+{ }^{2} \Delta(17)$ \\
\hline & $\mathrm{DFT}^{d}$ & 0 & 1.873 & & & & & 600 & \\
\hline & Expt. ${ }^{e}$ & 0 & 1.874 & & & & & 580 & \\
\hline \multirow[t]{4}{*}{$5 / 2$} & $\mathrm{MRCI}+\mathrm{Q}$ & 1019 & 1.905 & & & & & 604 & ${ }^{2} \Delta(100)$ \\
\hline & CCSD & 725 & 1.904 & & & & & & ${ }^{2} \Delta(100)$ \\
\hline & $\operatorname{CCSD}(\mathrm{T})$ & 747 & 1.905 & & & & & 594 & ${ }^{2} \Delta(100)$ \\
\hline & $\mathrm{DFT}^{d}$ & 1347 & 1.915 & & & & & 587 & \\
\hline \multicolumn{10}{|l|}{$\mathrm{HePtF}$} \\
\hline \multirow[t]{3}{*}{$1 / 2$} & $\mathrm{MRCI}+\mathrm{Q}$ & 0 & 1.884 & 1.835 & 2290 & 381 & $543(11)$ & $632(42)$ & ${ }^{2} \Sigma^{+}(74)+{ }^{2} \Pi(26)$ \\
\hline & CCSD & 0 & 1.881 & 1.828 & 2432 & & & & ${ }^{2} \Sigma^{+}(73)+{ }^{2} \Pi(27)$ \\
\hline & $\operatorname{CCSD}(\mathrm{T})$ & 0 & 1.881 & 1.828 & 2482 & & $560(13)$ & $639(65)$ & ${ }^{2} \Sigma^{+}(71)+{ }^{2} \Pi(29)$ \\
\hline \multirow[t]{3}{*}{$3 / 2$} & MRCI+Q & 500 & 1.866 & 1.873 & 2002 & 433 & $498(7)$ & $637(48)$ & ${ }^{2} \Pi(81)+{ }^{2} \Delta$ (19) \\
\hline & CCSD & 401 & 1.864 & 1.861 & 2143 & & & & ${ }^{2} \Pi(81)+{ }^{2} \Delta(19)$ \\
\hline & $\operatorname{CCSD}(\mathrm{T})$ & 76 & 1.862 & 1.860 & 2229 & & $524(6)$ & $645(75)$ & ${ }^{2} \Pi(82)+{ }^{2} \Delta$ \\
\hline \multirow[t]{3}{*}{$5 / 2$} & $\mathrm{MRCI}+\mathrm{Q}$ & 546 & 1.901 & 1.801 & 2770 & 69 & $589(14)$ & $638(30)$ & ${ }^{2} \Delta(100)$ \\
\hline & CCSD & 326 & 1.899 & 1.797 & 2832 & & & & ${ }^{2} \Delta(100)$ \\
\hline & $\operatorname{CCSD}(\mathrm{T})$ & 246 & 1.900 & 1.798 & 2807 & & $597(20)$ & $641(45)$ & ${ }^{2} \Delta(100)$ \\
\hline
\end{tabular}

${ }^{a}$ Without corrections. ${ }^{b}$ Numbers in parentheses are IR intensities ( $\left.\mathrm{km} / \mathrm{mole}\right)$. The quantum numbers $v_{1}, v_{2}$, and $v_{3}$ refer to bending, He-Pt stretching,

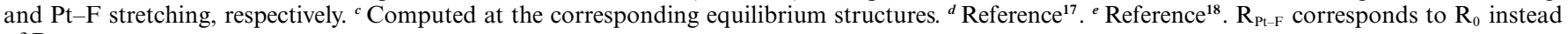
of $\mathrm{R}_{\mathrm{e}}$.

\section{Results and discussion}

To estimate the accuracy of the calculation methods for $\mathrm{HePtF}$, the low-lying spinor states of $\mathrm{PtF}$ were first calculated at the same levels of theory. By using four-component relativistic density functional theory (DFT), Liu and Franke ${ }^{17}$ predicted that the ground state of $\mathrm{PtF}$ is $\Omega=3 / 2$, and is different from that of $\mathrm{PtH}$ $(\Omega=5 / 2)$. This was further confirmed by a very recent microwave spectroscopy study. ${ }^{18}$ The published results as well as ours of PtF are summarized in Table 1.

It can be seen that the $\Omega=5 / 2$ state is ${ }^{2} \Delta$ dominant, and both $\Omega=1 / 2$ and $3 / 2$ states are mixtures of ${ }^{2} \Sigma^{+}+{ }^{2} \Pi$ and ${ }^{2} \Pi+{ }^{2} \Delta$, respectively. Among these states, $\operatorname{CCSD}(\mathrm{T})$ obtains $\Omega=3 / 2$ as the ground state, being in agreement with the experimental and the DFT results, whereas MRCI+Q and CCSD erroneously give $\Omega=$ $1 / 2$ as the ground state. Obviously the higher-order correlations which are absent in MRCI+Q and CCSD are very important for the small energy gap between the $\Omega=1 / 2$ and $3 / 2$ states of PtF. Nevertheless, the computed bond distances and vibrational frequencies by all the three methods are in good agreement with the DFT and available experimental values. It is expected that a similar accuracy can be achieved also for HePtF.

The spectroscopic constants of $\mathrm{HePtF}$ are listed in Table 1 as well. We can see that all of the methods support $\Omega=1 / 2$ as the ground state. However, for the first excited state, MRCI+Q and $\operatorname{CCSD}(\mathrm{T})$ support $\Omega=3 / 2$, being inconsistent with CCSD. Since MRCI+Q or CCSD(T) is more accurate than CCSD in the reference wavefunction or correlations, we can conclude that the first excited state should be $3 / 2$. Similar to $\mathrm{HeCuF}$ and $\mathrm{HeAuF}^{3}$ all the three spinor states of $\mathrm{HePtF}$ are computed to be linear, which are supported by the real bending vibrational frequencies $\left(v_{1}\right)$. Compared with $\mathrm{PtF}$, the Pt-F distances in $\mathrm{HePtF}$ are significantly shortened by about $0.01 \AA$ (for $1 / 2$ and $3 / 2$ ) or $0.005 \AA(5 / 2)$. This phenomenon has also been found in $\mathrm{HeCuF}$ and $\mathrm{HeAuF}^{3}$ as well as other analogues. ${ }^{3}$ The short HePt distances (1.80 1.87 $\mathrm{A}$ ), the large He-Pt stretching frequencies $\left(500 \sim 600 \mathrm{~cm}^{-1}\right)$, and the considerable binding energies of helium (2000 $2800 \mathrm{~cm}^{-1}$; uncorrected) also can be found from our results, which indicate that there is relatively strong interaction between the helium and platinum atoms in HePtF.

Since the binding energies are not sufficiently large, corrections must be included at the fixed geometries via the zero point energy (ZPE), basis set superposition error (BSSE), ${ }^{19}$ and CBSE, ${ }^{12}$ as shown in Table 2. In the CCSD(T) calculations of HePtF, the bending modes in ZPE were taken from the results of MRCI+Q since the Hartree-Fock method can not describe the excited states properly. The basis sets used here are quite large, so both the BSSE and the CBSE corrections are quite small, and ZPE contributes the dominant corrections for the binding energies. After including these corrections, it can be seen in Table 2 that the states still have considerable binding energies, being $1400 \sim 2500 \mathrm{~cm}^{-1}$ by the $\mathrm{MRCI}+\mathrm{Q}$ and $\mathrm{CCSD}(\mathrm{T})$ methods.

For comparison, we may estimate the lower limit of the van der Waals distance and the standard covalent distance for the $\mathrm{He}-\mathrm{Pt}$ bond by using the atomic van der Waals radius $\left(\mathrm{R}_{\mathrm{vdW}}\right){ }^{20}$ ionic radius $\left(\mathrm{R}_{\mathrm{ion}}\right){ }^{21}$ and covalent radius $\left(\mathrm{R}_{\mathrm{cov}}\right)^{22}$ with the following 
Table 2 Corrected binding energies of $\operatorname{HePtF}\left(\mathrm{cm}^{-1}\right)$

\begin{tabular}{|c|c|c|c|c|c|c|}
\hline \multirow[b]{2}{*}{ Methods $^{a}$} & \multicolumn{2}{|l|}{$1 / 2$} & \multicolumn{2}{|l|}{$3 / 2$} & \multicolumn{2}{|l|}{$5 / 2$} \\
\hline & $\mathrm{MRCI}+\mathrm{Q}$ & $\operatorname{CCSD}(\mathrm{T})$ & $\mathrm{MRCI}+\mathrm{Q}$ & $\operatorname{CCSD}(\mathrm{T})$ & $\mathrm{MRCI}+\mathrm{Q}$ & $\operatorname{CCSD}(\mathrm{T})$ \\
\hline uncorrected & 2290 & 2482 & 2002 & 2229 & 2770 & 2807 \\
\hline $\mathrm{ZPE}(\mathrm{PtF})$ & +293 & +295 & +295 & +297 & +302 & +297 \\
\hline $\mathrm{ZPE}(\mathrm{HePtF})^{b}$ & -969 & -981 & -1000 & -1017 & -683 & -688 \\
\hline BSSE & -36 & -43 & -35 & -41 & -41 & -48 \\
\hline CBSE & +166 & +123 & +133 & +83 & +145 & +118 \\
\hline Total & 1745 & 1877 & 1395 & 1551 & 2493 & 2486 \\
\hline
\end{tabular}

${ }^{a}$ ZPE, BSSE, and CBSE represent the zero point energy, basis set superposition error, and complete basis set extrapolation, respectively. ${ }^{b}$ Bending modes are taken from the MRCI+Q results.

Table 3 Mulliken charges for the scalar states of $\mathrm{PtF}$ and HePtF, computed by the MRAQCC method at the $\operatorname{CCSD}(\mathrm{T})$ optimized structures

\begin{tabular}{lllll}
\hline & State & Pt & F & He \\
\hline $\mathrm{PtF}$ & $1 / 2\left({ }^{2} \Sigma^{+}\right)$ & +0.51 & -0.51 & \\
& $3 / 2\left({ }^{2} \Pi\right)$ & +0.48 & -0.48 & \\
$\mathrm{HePtF}$ & $5 / 2\left({ }^{2} \Delta\right)$ & +0.51 & -0.51 & \\
& $1 / 2\left({ }^{2} \Sigma^{+}\right)$ & +0.46 & -0.52 & +0.06 \\
& $3 / 2\left({ }^{2} \Pi\right)$ & +0.42 & -0.48 & +0.06 \\
& $5 / 2\left({ }^{2} \Delta\right)$ & +0.47 & -0.53 & +0.06 \\
\hline
\end{tabular}

formulae, ${ }^{23} \mathrm{R}_{\mathrm{vdw}}(\mathrm{He}-\mathrm{Pt})=\mathrm{R}_{\mathrm{vdw}}(\mathrm{He})+\mathrm{R}_{\mathrm{ion}}\left(\mathrm{Pt}^{+}\right)=1.40+1.24=$ $2.64 \AA$, and $\mathrm{R}_{\text {cov }}(\mathrm{He}-\mathrm{Pt})=\mathrm{R}_{\text {cov }}(\mathrm{He})+\mathrm{R}_{\text {cov }}(\mathrm{Pt})=0.46+1.23=$ $1.69 \AA$.

For different states the optimized $\mathrm{R}_{\mathrm{He}-\mathrm{Pt}}$ values range in 1.80 1.87 $\AA$ (see Table 1) by different methods, being much shorter than $\mathrm{R}_{\mathrm{vdw}}(\mathrm{He}-\mathrm{Pt})$ and closer to $\mathrm{R}_{\mathrm{cov}}(\mathrm{He}-\mathrm{Pt})$.

At the structures optimized by $\operatorname{CCSD}(\mathrm{T})$ with $\mathrm{SOC}$, the Mulliken charges were calculated for the dominant scalar state of each spinor state by the internally contracted multi-reference average quadratic coupled-cluster method (MRAQCC), ${ }^{24,25}$ which can be looked on as an approximation to multi-reference CCSD. The computed Mulliken charges are listed in Table 3. For the fluorine atom, we see that there is no significant change in the Mulliken charges, and a small electron transfer (about 0.06 charge for all the three states) takes place from the $\mathrm{He}$ atom to the $\mathrm{Pt}$ atom. Again, this implies the interaction between $\mathrm{He}$ and Pt.

Although the interaction between $\mathrm{He}$ and $\mathrm{Pt}$ is quite strong according to the above analysis, it does not necessarily imply a covalent $\mathrm{He}-\mathrm{Pt}$ bond. To identify the covalent bond, the negative Laplacian $\left[\nabla^{2} \rho(r)\right]$ at the critical point $\left(r_{\mathrm{c}}\right)$ is often used as an indicator, but fails for some covalent molecules (e.g., the Laplacian is positive for $\mathrm{BeO}^{4 \mathrm{a}}$ and $\mathrm{F}_{2}{ }^{26}$ ). So it is a necessary condition instead of a sufficient one. ${ }^{4 a}$ Because of this reason, Cremer and Kraka ${ }^{26}$ suggested the use of $\mathrm{H}(\mathrm{r})=\mathrm{G}(\mathrm{r})+\mathrm{V}(\mathrm{r})$ (local kinetic energy density and local potential energy density, respectively) for this problem. Here we use a modified version of Bader's AIMPAC program ${ }^{27}$ to analyze the electron density distribution of the ground state. The wavefunction file was generated at the MRAQCC level of theory and the format was then converted by our Molden2WFN program. Because of the limitation of the AIMPAC program, the all-electron relativistic ANO-RCC basis set $^{28}$ with 4-zeta quality was adopted for all the atoms. At the $r_{\mathrm{c}}$ point between $\mathrm{He}$ and $\mathrm{Pt}$, it is found that the $\nabla^{2} \rho\left(r_{\mathrm{c}}\right)$ values are positive $(13.57,10.84$, and $14.27 \mathrm{e} / \AA^{5}$ for the scalar states ${ }^{2} \Sigma^{+},{ }^{2} \Pi$, and ${ }^{2} \Delta$, respectively) whereas the $\mathrm{H}\left(r_{\mathrm{c}}\right)$ values are negative but very small $(-0.03,-0.08$, and -0.09 Hartree $/ \AA^{3}$ ). Thus HePtF is a strong van der Waals complex with small covalent interaction. Perhaps it is difficult to define a clear borderline between strong van der Waals and weak covalent bonds.

In order to make clear the origin of the He-Pt bond, the natural bond orbital (NBO) analysis based on DFT(PBE0)/VQZ was carried out by the NBO program ${ }^{29}$ via the PC-GAMESS/Firefly ${ }^{30}$ interface. Since PC-GAMESS/Firefly cannot treat $h$-functions in the RECP and basis set, another RECP with 60 core electrons ${ }^{31}$ was used for $\mathrm{Pt}$, and the valence basis set were recontracted accordingly. Similar to the case of $\mathrm{HeAuF},{ }^{3} \mathrm{NBO}$ shows that the dominant interaction comes from the charge transfer from $\mathrm{He} 1 \mathrm{~s}$ to a weakly-occupied antibonding orbital between $\mathrm{F} 2 \mathrm{p}_{\sigma}$ and $\mathrm{Pt}$ $6 \mathrm{~s}$ with a certain amount of $\mathrm{Pt} 5 \mathrm{~d}_{\sigma}$. In addition, the analysis also gives a NBO charge of +0.07 on the helium atom, being very close to the Mulliken one ( $c f$. Table 3), and a Wiberg bond index of 0.15 for the He-Pt bond.

\section{Conclusions}

The lowest three spinor states $(\Omega=1 / 2,3 / 2$, and $5 / 2)$ of the linear $\mathrm{HePtF}$ molecule have been investigated by highly accurate relativistic ab initio methods. All evidence, including the structures, vibrational frequencies, binding energies, and Mulliken charges, show that the He-Pt bond is much stronger than typical van der Waals interactions. However, no covalent contributions between $\mathrm{He}$ and $\mathrm{Pt}$ atoms can be found from the topological analysis of the electron density distribution, so $\mathrm{HePtF}$ is a kind of uncommonly strong van der Waals complex, similar to the case of $\mathrm{HeCuF}^{3}$ $\mathrm{HeAuF},{ }^{3}$ and $\mathrm{HeBeO}{ }^{4}$

\section{Acknowledgements}

This research has been supported by Welch Foundation Grant No. F-100.

\section{Notes and references}

1 N. Bartlett, Proc. Chem. Soc., 1962, 218.

2 R. B. Gerber, Annu. Rev. Phys. Chem., 2004, 55, 55.

3 W. Zou, Y. Liu and J. E. Boggs, Chem. Phys. Lett., 2009, 482, 207.

4 (a) G. Frenking, W. Koch, J. Gauss and D. Cremer, J. Am. Chem. Soc., 1988, 110, 8007; (b) S. Borocci, N. Bronzolino and F. Grandinetti, Chem. Phys. Lett., 2005, 406, 179; (c) T. Takayanagi, H. Motegi, Y. Taketsugu and T. Taketsugu, Chem. Phys. Lett., 2008, 454, 1; (d) H. Motegi, A. Kakizaki, T. Takayanagi, Y. Taketsugu, T. Taketsugu and M. Shiga, Chem. Phys., 2008, 354, 38. 
5 K. Andersson and B. O. Roos, Chem. Phys. Lett., 1992, 191, 507. 6 W. Zou and W. Liu, J. Chem. Phys., 2006, 124, 154312.

7 D. Figgen, K. A. Peterson, M. Dolg and H. Stoll, J. Chem. Phys., 2009, 130, 164108.

8 L. F. Pacios and P. A. Christiansen, J. Chem. Phys., 1985, 82, 2664.

9 T. H. Dunning Jr., J. Chem. Phys., 1989, 90, 1007.

10 P. J. Knowles and H.-J. Werner, Theor. Chim. Acta, 1992, 84, 95.

11 D. E. Woon and T. H. Dunning Jr., J. Chem. Phys., 1994, 100, 2975.

12 A. Halkier, T. Helgaker, P. Jørgensen, W. Klopper, H. Koch, J. Olsen and A. K. Wilson, Chem. Phys. Lett., 1998, 286, 243.

13 P. J. Knowles, C. Hampel and H.-J. Werner, J. Chem. Phys., 1993, 99, 5219.

14 P. J. Knowles, C. Hampel and H.-J. Werner, J. Chem. Phys., 2000, 112, 3106(erratum).

15 A. Berning, M. Schweizer, H.-J. Werner, P. J. Knowles and P. Palmieri, Mol. Phys., 2000, 98, 1823.

16 H.-J. Werner, P. J. Knowles, R. Lindh, F. R. Manby, M. Schütz and others, MOLPRO, version 2008.1, a package of ab initio programs.

17 W. Liu and R. Franke, J. Comput. Chem., 2002, 23, 564.

18 T. Okabayashi, T. Kurahara, E. Y. Okabayashi and M. Tanimoto, Microwave spectroscopy of platinum monofluoride PtF, the 9th Symposium on Molecular Spectroscopy, University of Toyama, Toyama, Japan, May 16, 2009 (in Japanese). See: http://www.sci.u-toyama.ac.jp/ phys/4ken/molsp9/abst/t24.pdf.

19 S. F. Boys and F. Bernardi, Mol. Phys., 1970, 19, 553.
20 P. Pyykkö, Chem. Rev., 1997, 97, 597.

21 N. Chen, W. Lu, J. Yang and G. Li, Support Vector Machine in Chemistry, World Scientific, Singapore, 2004.

22 P. Pyykkö and M. Atsumi, Chem.-Eur. J., 2009, 15, 186.

23 J. M. Thomas, N. R. Walker, S. A. Cooke and M. C. L. Gerry, J. Am. Chem. Soc., 2004, 126, 1235.

24 P. G. Szalay and R. J. Bartlett, Chem. Phys. Lett., 1993, 214, 481.

25 H.-J. Werner and P. J. Knowles, Theor. Chim. Acta, 1991, 78, 175.

26 D. Cremer and E. Kraka, Angew. Chem., Int. Ed. Engl., 1984, 23, 627.

27 R. F. W. Bader, AIMPAC, 1994. See: http://www.chemistry.mcmaster. ca/aimpac/. The program was modified in order to use $g$ basis functions.

28 (a) P.-O. Widmark, P.-Å. Malmqvist and B. O. Roos, Theor. Chim. Acta, 1990, 77, 291; (b) B. O. Roos, R. Lindh, P.-Å. Malmqvist, V. Veryazov and P.-O. Widmark, J. Phys. Chem. A, 2004, 108, 2851; (c) B. O. Roos, R. Lindh, P.-Å. Malmqvist, V. Veryazov and P.-O. Widmark, J. Phys. Chem. A, 2005, 109, 6575; (d) See http://www.teokem.lu.se/ molcas/basis/70/list.html.

29 E. D. Glendening, J. K. Badenhoop, A. E. Reed, J. E. Carpenter, J. A. Bohmann, C. M. Morales and F. Weinhold, NBO 5.G, Theoretical Chemistry Institute, University of Wisconsin, Madison, WI, 2004.

30 A. A. Granovsky, PC GAMESS/Firefly, Version 7.1.F, 2009. See: http://classic.chem.msu.su/gran/gamess/index.html.

31 R. B. Ross, J. M. Powers, T. Atashroo, W. C. Ermler, L. A. LaJohn and P. A. Christiansen, J. Chem. Phys., 1990, 93, 6654. 